\title{
Carriers that count
}

\author{
An exactly solvable model for superconductivity includes two crucial features of the cuprates and sheds light on \\ unexplained experiments.
}

\section{Jan Zaanen}

$\mathrm{T}$ he Bardeen-Cooper-Schrieffer (BCS) theory of superconductivity ${ }^{1}$ was a landmark achievement of quantum many-body physics in the 1950s. For some time, the community thought that superconductivity was understood, but the subject was revived in the late 1980s with the discovery of a different form of superconductivity with high critical temperature in copper oxides. Since then, high-temperature superconductivity has earned the reputation of being the biggest conundrum of condensed matter physics ${ }^{2}$, in part because the non-superconducting state from which the superconductivity appears is very different from the BCS paradigm. Writing in Nature Physics, Philip Phillips and collaborators now take a conceptual step forward by finding an exactly-solvable model that captures important features of the normal state and can allow superconductivity.

The main focus of theoretical work in this field is to explain why the critical temperature is high, but over time it turned into a treasure trove for intriguing - if poorly understood - physics. The late Philip Anderson deserves credit for explaining the origin of this 'strangeness'. It arises from the fact that the basic materials from which high-temperature superconductors are made are Mott insulators: the electrons in the copper oxide repel each other very strongly and when there are an integer number of electrons per unit cell the electronic equivalent of a traffic jam is formed. This is turned into a superconducting metal by chemical doping, which is like electronic 'stop-and-go' traffic: the 'Mottness' condition ${ }^{4}$. This forms a fundamental obstruction for the system to renormalize into a Fermi-liquid-based BCS superconductor ${ }^{5}$, leaving room for the strange stuff to take over.

In contrast, the regular BCS superconductivity appears as an instability of the Fermi surface in a normal metal. This means that the BCS superconductor submits to the Luttinger and Leggett counting theorems. The Luttinger counting theorem states that the volume enclosed by the

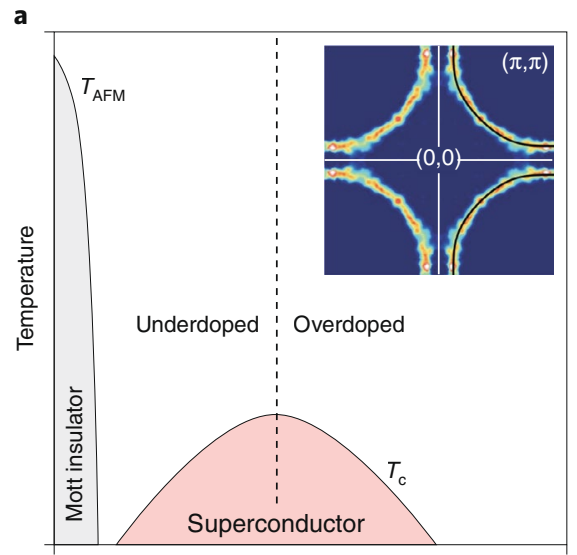

Doping

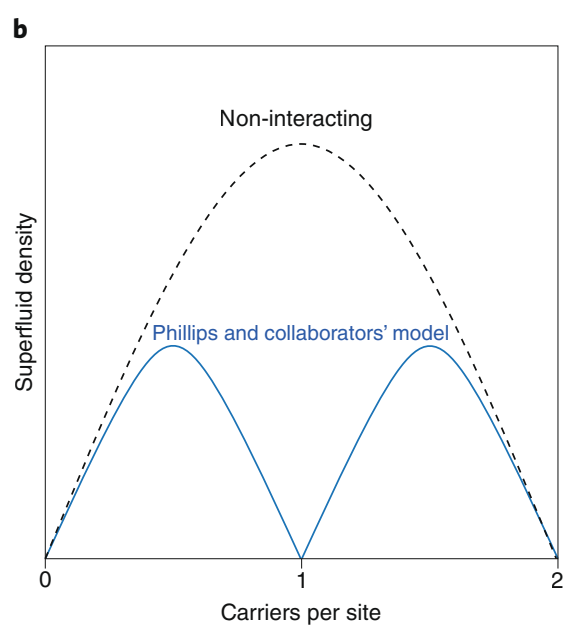

Fig. 1 | Phase diagram and superfluid density. a, Schematic phase diagram of the cuprates. The vertical dotted line indicates the doping at which the critical temperature, $T_{c}$, is the highest ${ }^{2}$. The low doping phase is a Mott antiferromagnet (AFM), from which superconductivity emerges with increasing doping. The inset shows an example Fermi surface from ARPES measurements ${ }^{10}$. $\mathbf{b}$, Schematic of the prediction for the superfluid density as a function of electron density from Phillips and collaborators' model ${ }^{3}$ (blue line) and for a non-interacting theory (black dotted line).

Fermi surface in momentum space equals the density of electrons, while the Leggett one says that in the effective Galilean continuum the zero-temperature superfluid density has to be equal to the normal-state density. These imply together that the Fermi surface determines the superfluid density $^{6}$. Both theorems are rooted in the concept of adiabatic continuity: these counting theorems are not only true for a non-interacting Fermi gas, but continue to be valid regardless of the strength of the interactions as long as the overlap of the states characterizing the Fermi gas and interacting ground state is finite.

However, for the cuprates (the typical phase diagram of which is shown in Fig. 1a), the situation is more murky. The critical temperature of the superconducting phase has a dome shape, and the doping level at which the maximum occurs marks the crossover from the underdoped to the overdoped regime ${ }^{1,2}$. These have quite different properties, and on the overdoped side, ARPES experiments have shown sharp normal-state quasiparticles that form a genuine Fermi surface 7 . But a surprising experimental result interfered with this picture of the overdoped regime as a well-behaved Fermi liquid. In a Fermi-liquid-based BCS superconductor, the superfluid density (the density of carriers in the supercurrent) has to be of the order of the large microscopic electron density according to the Legget theorem - which is in turn roughly independent of the doping. This superfluid density was directly measured by magnetization experiments over the whole overdoped regime, revealing that it is much smaller than the BCS expectation ${ }^{8}$. This is the apparent paradox ${ }^{6}$ : how to reconcile the good Fermi surface revealed by the ARPES and the violation of the counting theorems as revealed by the superfluid density.

The solution departs from a model ${ }^{9}$ that has been lying dormant for 23 years, where a metal is characterized by a Fermi surface 
but simultaneously exploits a mathematical loophole to embed the essence of Mottness, the fact that the metal is a doped Mott insulator. The model appears to resolve the conundrum of being a non-Fermi liquid with a Fermi surface in a seemingly unique way. It is very simple: the Pauli-principle counting of the Fermi gas that mandates one electron per spin and site, and the Mottness counting of only one electron per site, can be reconciled by a large contact (Hubbard) interaction that acts only between electrons that have the same momentum. This interaction is microscopically unphysical it is highly non-local in real space - but it does quite well in capturing the qualitative effects of the Mottness, for example in the form of representing upper and lower Hubbard bands in spectral functions $s^{3,9}$.

Phillips and collaborators take this a step further by adding a BCS-style attractive interaction to the model. The result is that it shares qualitative features with BCS $^{3}$ : superconductivity sets in by Cooper instability at a thermal transition that opens a superconducting gap. By construction, the model has a Fermi surface, but this will enclose a different volume in momentum space violating the Luttinger theorem: the Hubbard interaction forces doubly occupied momentum space states into two singly occupied ones in parts of momentum space. Similar logic applies to the Leggett theorem and gives a relation between the normal electron density and superfluid density (shown in Fig. 1b) that is completely different from BCS, showing a very strong suppression of the superfluid density near the density of one electron per site where the Mott insulator appears, reminiscent of the experimental observations.

This construction has at the least the merit of teaching us how to think outside the box of BCS theory, which is much desired given the apparent paradox of the superfluid density $^{6}$. But perhaps we should actually take it more literally. Adiabatic continuity has a remarkable capacity to connect seemingly unrelated entities - for instance, in the ${ }^{3} \mathrm{He}$ liquid the macroscopic free Fermi-gas fixed point is adiabatically continued to the dense van der Waals liquid on the microscopic scale. The barely explored phenomenology of Phillips and collaborators' model may well have more surprises in store ${ }^{3}$ that - by confrontation with reality - may eventually decide if there is a similar continuity between the microscopic Mottness and this non-Fermi liquid with Fermi-surface fixed point.

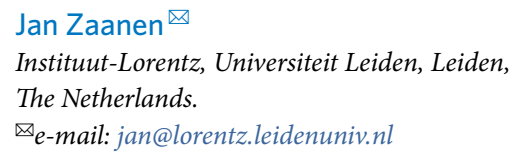

Published online: 27 July 2020

https://doi.org/10.1038/s41567-020-0979-5

References

1. Bardeen, J., Cooper, L. N. \& Schrieffer, J. R. Phys. Rev. 106, 162-164 (1957).

2. Keimer, B., Kivelson, S. A., Norman, M. R., Uchida, S. \& Zaanen, J. Nature 518, 179-186 (2015).

3. Phillips, P. W., Yeo, L. \& Huang, E. W. Nat. Phys. https://doi. org/10.1038/s41567-020-0988-4 (2020).

4. Phillips, P. W. Rev. Mod. Phys. 82, 1719-1742 (2010).

5. Anderson, P. W. The Theory of Superconductivity in the High-T

Cuprate Superconductors (Princeton Univ. Press, 1997).

6. Zaanen, J. Nature 536, 282-283 (2016).

7. Chen, S. D. et al. Science 366, 1099-1102 (2019).

8. Bozovic, I., He, X., Wu, J. \& Bollinger, A. T. Nature 536, 309-311 (2016).

9. Hatsugai, Y. \& Kohmoto, M. J. Phys. Soc. Jpn 61, 2056-2069 (1992).

10. Platé, M. et al. Phys. Rev. Lett. 95, 077001 (2005).

\title{
Facts are relative
}

\author{
The discussion of the quantum mechanical Wigner's friend thought experiment has regained intensity. Recent \\ theoretical results and experimental tests restrict the possibility of maintaining an observer-independent notion of \\ measurement outcomes.
}

\section{Časlav Brukner}

n 1983, Daniel Patrick Moynihan, an American politician, sociologist and diplomat, wrote that "Everyone is entitled to his own opinion, but not his own facts". As much as this may be applied to politics, in quantum mechanics, the objectivity of facts or 'observed events' in the sense that they exist absolutely - and not relative to a particular observer - has recently been challenged in a series of works ${ }^{1-4}$ that build on the Wigner's friend thought experiment ${ }^{5}$. First conceived by physicist Eugene Wigner in 1961, the scenario results in a situation where two observers seem to experience different facts. Writing in Nature Physics, Kok-Wei Bong and co-workers ${ }^{6}$ have now rigorously proved and experimentally demonstrated that a set of plausible assumptions, together with the assumption that an observed event is objective and not relative to anything or anyone, contradicts quantum mechanical predictions and experimental data. The result can be interpreted to imply that in quantum physics, observers are indeed entitled to their own facts.

In the original Wigner's friend thought experiment, an observer in an isolated laboratory - the friend - performs a measurement on a quantum system in an equal superposition of two states. She randomly obtains one of two possible outcomes and updates her system's state to match the observed outcome. The updated state can be verified by repeating the measurement. Meanwhile, a 'superobserver' outside the laboratory - Wigner describes his friend, the laboratory and her system as a large, composite quantum system. He is equipped with instruments of unprecedented precision, which enable him to ascertain the quantum state for the whole laboratory. Most importantly, his measurement that confirms the state does not assign a well-defined value to the outcome of the friend's observation. From Wigner's point of view, his friend exists in a coherent superposition, entangled with the outcome of her measurement. This apparently contradicts the friend's description. Reconciling these two perspectives is at the core of the Wigner's friend paradox. 